\title{
Memoria de rostros y reconocimiento emocional: generalidades teóricas, bases neurales y patologías asociadas
}

\author{
Face memory and emotional recognition: Theory, \\ neural substrates and related pathologies
}

\author{
Yunier Broche Pérez ${ }^{1}$ \\ Mirelly Rodríguez Almeida² \\ Universidad Central "Marta Abreu" de Las Villas, Cuba
}

\author{
Erislandy Omar Martínez ${ }^{3}$ \\ Universidad de Oriente, Cuba
}

\begin{abstract}
Resumen. La memoria de rostros y el reconocimiento emocional son procesos de gran interés para la psicología contemporánea. Desde la aparición del modelo de Bruce y Young en 1986 se han realizados importantes avances en la comprensión de los mecanismos que determinan estos procesos; para ello los investigadores se han apoyado en herramientas como la neuroimagen funcional y el registro de potenciales evocados. Esto ha propiciado la existencia de un mayor conocimiento de la memoria de rostros y el reconocimiento de emociones, facilitando su utilización en diversas áreas aplicadas. Este trabajo sintetiza los principales avances en este campo y su importancia para la psicología contemporánea, transitando desde las distintas posturas teóricas existentes hasta las bases neurales implicadas en los procesos.
\end{abstract}

Palabras clave. Memoria de rostros, reconocimiento emocional, modelos teóricos, bases neurales, patologías asociadas.

Abstract. Face memory and emotional recognition are really important processes for contemporary psychology. Since Bruce and Young published their model of cognitive organization of face perception processes, in 1986, the knowledge about these two cognitive mechanisms has growth considerably. This knowledge has been enriched by the use of several approaches, for example, neuropsychological, cognitive, and the physiological one, using sometimes the presentation of pictures to recognition and neuroimaging techniques in other times. The knowledge reached in this field has been employed in different areas of psychological sciences like forensic psychology and neuropsychological rehabilitation, among others. The aim of this paper is to summarize the most significant facts reached in the field beginning with theory analysis of different approaches, and ending with the neuropsychological and neural substrates of each process.

Keywords. Face memory, emotional recognition, theory approaches, neural substrates, related pathologies.

\footnotetext{
${ }^{1}$ Yunier Broche Pérez, Facultad de Psicología, Universidad Central "Marta Abreu" de Las Villas. Dirección postal: Código postal 54830, Universidad Central "Marta Abreu” de Las Villas, Carretera de Camajuaní Km 51⁄2 Santa Clara, Villa Clara, Cuba. E-mail: yunierbp@uclv.edu.cu

${ }^{2}$ Mirelly Rodríguez Almeida, Facultad de Psicología, Universidad Central “Marta Abreu” de Las Villas. E-mail: mirely@uclv.edu.cu

${ }^{3}$ Erislandy Omar Martínez, Centro de Estudios de Neurociencia, Procesamiento de Imágenes y Señales, Universidad de Oriente. E-mail: omar@fie.uo.edu.cu
} 


\section{Introducción}

El rostro constituye uno de los primeros estímulos que recibimos al nacer y desde el punto de vista biológico, psicológico y social es el objeto visual de mayor significación para el ser humano (Lopera, 2000). Una cara es la clave más distintiva y ampliamente usada para determinar la identidad de una persona (Bruce \& Young, 1986). La información que nos brinda el rostro relacionada con el sexo, la edad, la raza y las expresiones faciales de una persona, es instantáneamente accesible a nosotros y facilita la comunicación interpersonal.

Para la comunidad científica ha resultado de interés determinar la forma en que la información proveniente de una cara es procesada. El rostro pertenece a una categoría visoperceptual especial (Lopera, 2000) y desempeña un rol central en el desarrollo de habilidades para la interacción social y el lenguaje. El procesamiento facial ha merecido la atención de diversos autores enfocados en determinar y explicar los distintos ámbitos de procesamiento que pueden estar implicados en el reconocimiento facial, basados en estudios clínicos experimentales y neuropsicológicos.

\section{Principales modelos que explican el procesamiento facial}

El procesamiento de rostros ha sido explicado a través de modelos donde se toma en cuenta los procesos perceptuales y cognitivos que están relacionados y describen los diferentes pasos que este involucra. Los principales modelos que serán descritos por su relevancia en el ámbito científico son: el Modelo Funcional para el Procesamiento de Rostros propuesto por Bruce y Young (1986) y el Modelo de la Distribución Neural para la Percepción de Rostros de Haxby, Hoffman y Gobbini (2000).

Modelo Funcional para el Procesamiento de Rostros. Este modelo defiende que nuestro cerebro tiene un sistema especializado en la identificación de caras, diferente al sistema de identificación de otros estímulos visuales. Bruce y Young (1986), dan énfasis a distintos procesos psicológicos relacionados con la identificación de las expresiones faciales o la identidad facial (Figura 1). Apuntan además que el procesamiento de la identidad del rostro es un proceso altamente elaborado que se fragmenta en diferentes sub-procesos o pasos: construcción del percepto facial, reconocimiento facial, activación de la memoria semántica relativa a las personas, acceso lexical y producción articulatoria (Lopera, 2000). Estas cuatro rutas separadas permiten derivar diferentes tipos de información.

El primero de estos pasos, conlleva la construcción del percepto visual de la codificación estructural de las características faciales, lo cual requiere un análisis simultáneo y en paralelo de diferentes tipos de información facial: la apariencia o patrón facial, la discriminación de las características particulares del rostro y su distribución espacial particular monoorientada que permite reconocer diferencias y semejanzas entre las caras y el análisis de las expresiones faciales y la lectura labio-facial (Bruce \& Young, 1986).

Cuando se construye el percepto visual del rostro se compara para lograr el reconocimiento facial que constituye el segundo paso. De esta forma se puede determinar si el rostro percibido es conocido o totalmente nuevo. Lo anterior se logra a partir de los almacenes de memorias de caras o nodos de identidad personal como también son conocidos (Broche \& Herrera, 2011).

El tercerproceso del modelo parte del reconocimiento del rostro y del sentimiento de familiaridad que se produce lo cual conlleva a la activación del nodo de identidad personal y al acceso a la información relativa a la persona. Este nodo es accesible a través de la vía del percepto facial de la persona y del percepto auditivo (Bruce \& Young, 1986).

El acceso lexical es el siguiente paso, donde se establece el vínculo entre los elementos precedentes y la producción articulatoria: se activa la representación verbal del objeto percibido que permite, finalmente, efectuar la acción lexical del acto de reconocimiento a través de la pronunciación del nombre (Broche \& Herrera, 2011).

Este modelo distingue, el procesamiento de caras familiares respecto al de las no familiares (GarcíaGarcía \& Cacho-Gutiérrez, 2004). La idea central 


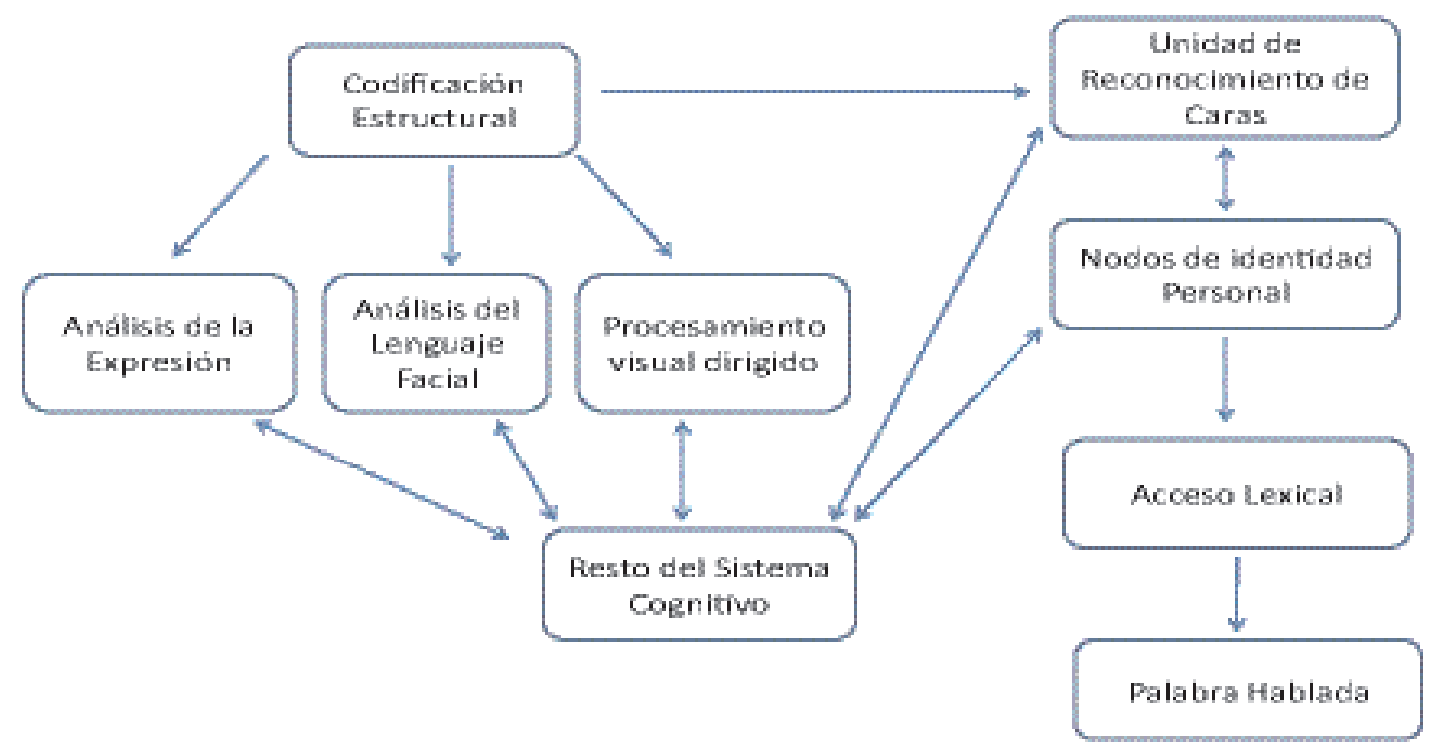

Figura 1. Modelo Funcional para el Procesamiento de Rostros de Bruce y Young.

que defiende es que el procesamiento facial consiste en un set de procesos independientes, por lo que el reconocimiento e identificación del rostro es independiente de la codificación de dimensiones de la cara como género o expresión.

Además el modelo ha sido ampliamente utilizado para entender el procesamiento facial porque ofrece un enfoque teórico contundente. Como limitación se le señala que los resultados que se obtienen con ciertos pacientes no parecen apoyar la distinción entre el reconocimiento de caras familiares y no familiares (García-García \& Cacho-Gutiérrez, 2004).

Modelo de la Distribución Neural para la Percepción de Rostros. A diferencia del modelo de Bruce y Young (1986), el modelo propuesto por Haxby y Gobbini (2000, 2002), abarca las bases anatomofuncionales que sostienen el proceso de reconocimiento de caras (Figura 2). Significa que, mientras Bruce y Young establecen una propuesta de modelamiento cognitivo, Haxby y Gobbini ofrecen los supuestos biológicos que sostienen el mecanismo.

Este modelo tiene como antecedente las observaciones realizadas a pacientes con daño cerebral focal con afectaciones en la habilidad de reconocer rostros. El mismo enfatiza en la distinción entre la representación de los aspectos invariantes de los rostros que permiten reconocer la identidad y la representación de aspectos variables que facilitan la interacción social. Esta propuesta muestra una estructura jerárquica que distingue un sistema central para el análisis visual de los rostros y otro para el proceso que permite dotar de significado la información que se obtiene de las caras (Haxby, Hoffman \& Gobbini, 2000).

El sistema doble, consiste en tres áreas bilaterales con una configuración anatómica que sugiere una organización jerárquica, donde la región occipital inferior provee el estímulo a la zona lateral fusiforme y a las regiones del surco temporal. A su vez, este sistema neural tiene extensiones hacia el sistema de percepción facial (Haxby, Hoffman \& Gobbini, 2000). El sistema neural para la atención espacial y la percepción, con las regiones cerebrales ubicadas en el surco intraparietal, más probablemente, en las áreas visual frontal, procesa la información proveniente de los rostros, como la mirada y la posición de la cabeza para dirigir la atención.

Haxby, Hoffman y Gobbini (2002) también hacen referencia al sistema neural para la comprensión verbal auditiva localizado en el giro temporal superior por su participación en el procesamiento de la lectura de los labios, en la extracción de información fonética. 


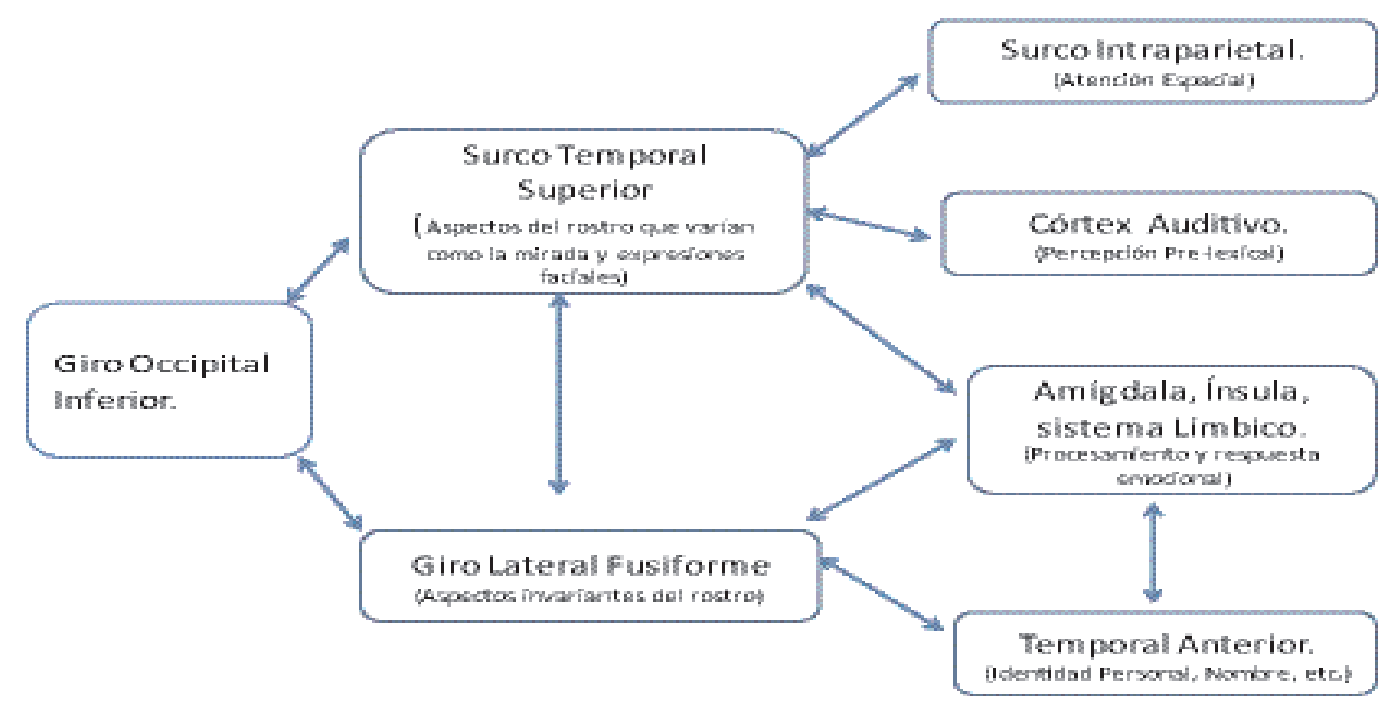

Figura 2. Modelo de la Distribución Neural para la Percepción de Rostros de Haxby.

Los sistemas neurales para representar conocimiento semántico biográfico en el lóbulo temporal anterior participan en la recuperación del nombre y otra información relacionada con el rostro. Estos autores también hacen referencia a los sistemas para el procesamiento de la emoción, que se hallan en la amígdala y la ínsula y que procesan la información con contenido emocional.

Los autores proponen además que muchas de las funciones perceptuales faciales se logran por la participación coordinada de múltiples regiones. El procesamiento de la información espacial que procede de la mirada y la posición de cabeza, involucra la participación coordinada de la región sensible a la percepción facial en el surco temporal superior y el sistema de atención espacial en el surco intraparietal (Haxby, Hoffman \& Gobbini, 2000). Por otra parte, la percepción de la expresión emocional involucra la participación coordinada de regiones para el análisis visual de expresión y las regiones que representan y producen las emociones (Broche \& Herrera, 2011).

De acuerdo con este modelo una función cognoscitivamente definida como la lectura de los labios, no involucra una región del cerebro especializada para esa función, sino que es la actividad convenida de las regiones las que posibilitan que la función se cumpla. Este planteamiento está relacionado con lo que Luria (1982) define como sistemas funcionales, por lo que estas mismas regiones también pueden participar en otras funciones actuando recíprocamente con otros sistemas. El modelo proporciona una descripción de subsistemas funcionales que son el fundamento de muchos aspectos de la cognición social.

Estos modelos han establecido las bases de las investigaciones que se desarrollan en la actualidad relacionadas con el procesamiento, memoria y reconocimiento de rostros. En las últimas dos décadas se han realizado numerosos estudios para encontrar la evidencia que respalde el funcionamiento a nivel cerebral del procesamiento de la información facial.

\section{Bases neurales del procesamiento de rostros}

La utilización de diferentes técnicas de neuroimagen como los potenciales evocados (ERP), magnetoencefalografía (MEG), tomografía de emisión de positrones (PET) y resonancia magnética funcional (fMRI); ha reportado amplia evidencia relacionada con el mecanismo neural especializado para las caras.

Los estudios magnetonecefalográficos (MEG) miden los campos magnéticos producidos por la 
actividad eléctrica en el cerebro (Duchaine \& Yovel, 2008). Se reportó en investigaciones realizadas por Liu, Harris y Kanwisher (2002), un componente de temprana aparición, que emerge a los $100 \mathrm{~ms}$ luego de la estimulación de un rostro. La M100 y M170 se reflejan ante la inversión del rostro, similar a la N170. La M170, a diferencia del N170 cuya amplitud se representa en ambos hemisferios, muestra mayor amplitud en el hemisferio derecho, lo cual sugiere cierta especialización de este hemisferio para el reconocimiento de los rostros (Henson, Rugg, Shallice, Josephs \& Dolan, 1999; Yovel, Levy, Grabowecky \& Paller, 2003).

Otro estudio realizado por Liu, Higuchi, Marantz y Kanwisher (2000) examina el rol del M100 y el M170 en la categorización de rostros (discriminación entre un rostro y una casa) y la identificación de rostros (discriminación entre dos rostros) determinando el nivel de ruido en esta tarea. Los resultados mostraron claramente que la M100 está relacionada con la categorización exitosa de rostros pero no con la identificación, mientras que la M170 si se relaciona con la realización exitosa de ambas tareas.

Harris y Nakayama (2007), examinaron el efecto de la repetición de categorías (casas y rostros) obteniendo que el M170 muestra un patrón de repetición solo cuando el tiempo de presentación entre el primer y segundo estímulo es inferior a los $400 \mathrm{~ms}$. Esto indica que la M170 se genera en estados iniciales del procesamiento de los rostros y que desaparece cuando la exposición se extiende por tiempos superiores a los $400 \mathrm{~ms}$.

Los resultados aportados por otras investigaciones muestran la intención de algunos autores de localizar en áreas cerebrales específicas la actividad relacionada con el reconocimiento facial. En este grupo se encuentran Kanwisher, McDermott y Chun (1997), quienes aseveran que la percepción de la cara evoca actividad en una región bilateral en el giro fusiforme lateral, que es mayor que la evocada por la percepción de objetos sin caras y de estímulos controlados. Esta región en particular, ha sido nombrada Área Facial Fusiforme (FFA).
En oposición a este planteamiento, Gauthier, Behrmann y Tarr (1999) han defendido que esta región se relaciona con la especialización visual y no sólo con el reconocimiento de la cara. Este autor propone que esta región puede participar en la percepción de otras categorías de los objetos, especialmente cuando esos objetos se reconocen en un nivel inferior de forma individual y no a nivel de categoría. Asimismo afirma que los rostros activan estas regiones porque prácticamente todos somos expertos en el reconocimiento facial (Haxby, Hoffman, \& Gobbini, 2002).

Se han realizado además estudios de imágenes funcionales que destacan la participación del giro occipital inferior y del surco temporal superior. Siguiendo esta perspectiva, existe evidencia a través de la medición de los potenciales evocados, usando electrodos situados en la superficie cortical, que pacientes expuestos a cirugía cerebral para la epilepsia, muestran en el lóbulo temporal respuestas específicas a los estímulos faciales en estas mismas zonas. El polo temporal anterior de igual forma se activa durante la percepción de las caras (Haxby, Hoffman \& Gobbini, 2002).

La corteza orbitofrontal se asocia también a la percepción facial, investigaciones realizadas en macacos, permitieron identificar neuronas que responden a estímulos en esta región (Thorpe, Rolls, \& Maddison, 1983). Asimismo, Rolls (1996) hace referencia al papel de la corteza orbitofrontal en la evaluación de la información proveniente de los rostros que es importante en el reforzamiento social: expresión e identidad.

Como se puede apreciar, diversas investigaciones se han enfocado en descubrir los sistemas neurales que subyacen en el procesamiento de los rostros. El progreso tecnológico alcanzado ha permitido optimizar los resultados obtenidos, los cuales hasta el momento nos permiten concluir que no existe una región específica relacionada con el reconocimiento facial, solo evidencias de zonas que pueden confluir o no en una región cuya función no se reduce solamente al procesamiento facial. 


\section{Memoria de rostros}

Las tareas de reconocimiento se han estudiado frecuentemente en conjunto con la memoria de rostros. Las investigaciones enfocadas solo en la memoria de rostros son recientes e insuficientes. En la literatura especializada en el abordaje de procesos cognitivos, específicamente la memoria, no se explica este proceso, por lo cual los autores parecen asumir que la memoria de caras opera bajo los mismos mecanismos y posee el mismo substrato que el resto de las memorias (Broche \& Herrera, 2011).

La memoria de rostros es la habilidad que determina nuestro éxito en el reconocimiento de la identidad en la vida cotidiana. Diferenciar a una persona de otra es una de las primeras habilidades cognitivas del ser humano, pues se desarrolla desde los primeros meses de vida.

De acuerdo con Manzanero (2008) existe una tipología propuesta por Jacobyy Dallas, señalando que existen dos variantes: reconocimiento perceptivo y memoria de reconocimiento. El primero de ellos funciona a través de juicios de familiaridad donde se toma en consideración la información física. Por otra parte la memoria de reconocimiento coincide con el reconocimiento por identificación, que es el resultado de un proceso de elaboración e implica la toma de decisión y la recuperación del contexto en que se codificó la información (Manzanero, 2008).

Adolphs (2002), siguiendo esta idea, plantea que el reconocimiento requiere un conocimiento sobre el mundo y además un tipo de memoria específica. También añade que una de las formas más simples de reconocimiento es en efecto la llamada memoria del reconocimiento y podría involucrar simplemente la habilidad de contener alguna información acerca de las propiedades perceptuales tempranas de una imagen visual, necesarias para que pueda ser comparada con otra imagen. Esta forma de reconocimiento permite la diferenciación entre dos caras presentadas por separado.

Manzanero (2008) refiriéndose a este proceso de reconocimiento de personas señala que en él, las experiencias de memoria dan lugar a dos formas diferentes de realizar la tarea de identificación: por familiaridad, que implica una vía directa que no requiere de procesamiento consciente; y por identificación que es indirecta y precisa de un proceso de elaboración consciente. El proceso de reconocimiento se basa, de acuerdo a Manzanero, Grandes y Jódar (2009) en la comparación de la imagen de la cara que recuerda la persona con la que se le presenta y además la toma de decisión respecto a si se encuentra ante la misma persona u otra diferente.

Para determinar cómo se comporta la memoria de rostros en diferentes grupos etarios, Pozzulo y Lindsay (1998) realizaron una sistematización de estudios anteriores, donde la tarea se enfoca en la identificación de personas adultas. De esta forma concluyeron, luego de examinar una muestra bastante amplia, que los niños de preescolar realizaban significativamente menos identificaciones correctas que los adultos, a diferencia de los escolares de entre 5 y 6 años. En los niños mayores y adolescentes no se evidenciaron diferencias significativas respecto a los adultos; en general se observó que el rendimiento estaba más bajo en los niños mayores. La explicación de este resultado que respaldan los autores, aunque existe discusión al respecto, es que estos niños mayores (entre 9 y 10 años) emplean una estrategia de codificación basada en rasgos más que en un procesamiento holístico, aun cuando a esta edad los niños ya podrían ser capaces de procesar globalmente la información facial (Manzanero, Grandes, \& Jódar, 2009).

En una serie de experimentos realizados por Germine, Duchaine y Nakayama (2010) con rostros en posición derecha e invertidos informan que la memoria de reconocimiento de caras aumenta significativamente durante la madurez temprana. También encontraron que la edad cumbre para la de memoria de rostros (3034 años) difiere con respecto a las de la memoria del reconocimiento para los nombres y las caras invertidas (23-24 años). Finalmente, afirman que la memoria de rostros puede continuar mejorando más allá del punto que indica el modelo, incluso cuando otras formas de memoria de reconocimiento visual ya estén desarrolladas. Esta conclusión está sustentada en la idea 
de que el reconocimiento de las caras es una habilidad que las personas van adquiriendo y que ejecutan diariamente, por tanto la práctica cotidiana contribuye a desarrollar esta habilidad al máximo de las posibilidades individuales (Germine, Duchaine \& Nakayama, 2010).

Como se puede apreciar, estos autores no realizan una conceptualización del proceso de memoria de rostros, sino que se aproximan al fenómeno a través de la categoría reconocimiento y es que este proceso ha sido el centro de pocas investigaciones. Duchaine y Yovel (2008) luego de una revisión teórica y metodológica que realizan, definen la memoria de rostros como la capacidad de ingresar, registrar, almacenar y recuperar la información relativa a las caras.

Es imprescindible cuando se estudia la memoria de rostros tener en cuenta las características de las caras que permiten que este sea recordado; las diferencias entre las caras dependen de matices, porque en esencia los rostros son iguales. Como resultado del envejecimiento, proceso natural que ocurre en el ser humano, las caras sufren modificaciones a largo plazo y también a corto plazo con las expresiones faciales.

En la memoria de rostros intervienen también aspectos emotivos, por lo cual se recuerdan más unas caras que otras. Los factores raciales influyen de igual modo pues se reconocen y recuerdan mejor las caras de los individuos de la propia raza: una persona mayor de raza caucásica reconoce mejor caras de este tipo, peor caras del norte de Europa y mucho peor caras de sujetos de raza negra o asiáticos (Broche \& Herrera, 2011).

\section{Bases funcionales y neurales de la memoria de rostros}

Ballesteros (1999) se refiere a la memoria de rostros como una operación compleja, destacando entre las regiones cerebrales que intervienen a la corteza visual occipital para el acceso e interpretación de la imagen, regiones frontotemporales para la organización e identificación de rasgos, el hipocampo para acceder a los recuerdos relativos a las personas que vemos y los polos temporales para acceder al nombre.

Germine, Duchaine y Nakayama (2010) señalan que en estudios dirigidos a determinar los cambios relacionados con la edad que ocurren en la memoria de caras a través del empleo de neuroimágenes funcionales, reportan una activación en la corteza temporal ventral a diferencia de otras regiones cercanas.

Las personas tienen la capacidad de memorizar un gran número de caras en el transcurso de la vida. El proceso de reconocer un rostro antes visto parece simple porque generalmente se realiza de manera holística, sin necesidad de una descripción verbal, ni de un análisis consciente de las diversas características faciales. Sin embargo esta capacidad de memorizar rostros puede afectarse con una lesión cerebral localizada en una región particular de la corteza cerebral (Lopera, 2000).

El reconocimiento de rostros familiares se efectúa de manera instantánea, a partir de informaciones provenientes de la visión y de memorias adquiridas en el pasado. Cuando se afecta el reconocimiento visual de caras, en ausencia de deterioro intelectual y con agudeza visual conservada, estamos antes la presencia de un trastorno conocido como prosopagnosia. Esta es una perturbación poco común que se asocia a lesiones temporo-occipitales derechas, o frecuentemente bilaterales (Ardila \& Ostrosky, 2012). Es importante señalar que las personas que presentan este trastorno no han perdido la capacidad de reconocer la identidad de una persona: lo que han perdido es la conexión entre una cara concreta y una identidad determinada (Kandel, 2007).

En los pacientes que presentan prosopagnosia pueden estar alterados diferentes procesos perceptivos, de procesamiento asociativo o de la memoria. GarcíaGarcía y Cacho-Gutiérrez (2004) hacen referencia a dos tipos de prosopagnosia: aperceptiva, donde existe un déficit perceptivo asociado al problema de identificación de rostros y asociativa, que está más relacionada con una dificultad en el procesamiento asociativo y en el acceso, almacenamiento o recuperación de información memorística asociada a las caras. Estos investigadores afirman que una lesión cerebral unilateral generalmente derecha, podría también provocar el déficit y no necesariamente debe ser producto de una lesión cerebral bilateral. En conclusión se aprecia que la prosopagnosia es descrita como el extremo patológico del proceso 
del recuerdo de un rostro, mostrando la completa incapacidad para acceder a la información de un rostro con familiaridad previa.

\section{Reconocimiento emocional}

Ciertamente el rostro nos provee información relacionada con la identidad, pero a su vez se convierte en una ventana hacia el estado emocional de las personas. Las emociones desempeñan un rol fundamental para la experiencia, supervivencia y desarrollo humano, a través de ellas se va conformando la personalidad, así como la manera de percibir y sensibilizarnos ante el mundo (Cereceda, Pizarro, Valdivia, Ceric, Hurtado \& Ibáñez, 2010).

El estudio de las emociones ha generado diversas teorías dentro de la ciencia psicológica. Se reconoce que la teoría evolucionista, representada por Duchenne de Bologne y Darwin consta como uno de los primeros trabajos que abordan tal categoría. Estos autores plantean la hipótesis de la universalidad de la expresión facial de las emociones y de su carácter relevante para la supervivencia de los individuos (Molerio, 2004). La autora también plantea que existe un acuerdo en reconocer la importante función adaptativa que tienen las emociones en tanto ellas actúan como poderosos incentivadores y dinamizadores de la conducta.

Otra función que cumplen las emociones es la de organizar numerosos sistemas biológicos como la expresión facial, los músculos, el tono de voz, el sistema nervioso autónomo y el sistema endocrino, propiciando así, un entorno corporal óptimo para una respuesta eficaz (Levenson, Ascher, Goodkind, Mccarthy \& Werner, 2008). Es preciso señalar además la importante función social que desempeñan, pues facilitan la interacción entre las personas favoreciendo las relaciones interpersonales a través del reconocimiento adecuado de emociones en los otros, y de la posibilidad de comportarnos de acuerdo a ellas.

El reconocimiento de emociones es un componente central de las competencias emocionales y es una base fundamental para la empatía y la habilidad de interpretar las reacciones de las personas y predecir las conductas resultantes (Bänziger, Grandjean \& Scherer, 2009).
El reconocimiento de las emociones faciales, de acuerdo con Adolphs (2002) se obtiene a través de tres estrategias complementarias. La primera es la percepción y depende de la activación de la corteza visual en respuesta a las características geométricas del rostro, lo que posibilita la vinculación del estímulo con la categoría específica de los rostros y a la vez detectar sus características elementales (edad, género). El segundo paso es el propio reconocimiento y depende del funcionamiento integrado de distintas áreas corticales. Este reconocimiento conlleva el análisis de los rasgos faciales que revelan emoción de manera más sobresaliente: los ojos y la boca. Es importante señalar que la información que se obtiene de la observación del rostro, se integra con datos provenientes de la memoria: experiencias pasadas o el conocimiento teórico de las emociones. Finalmente, a través del tercer mecanismo, el cerebro logra identificar las emociones faciales, pues se produce una activación en las zonas de la corteza motora, lo que provoca un efecto de simulación o representación interna de las posturas observadas y genera el estado emocional que se cree correspondiente al observado. Estas estrategias que intervienen en el reconocimiento emocional, deben ser analizadas, como un continuo, siendo necesaria la actuación de todas para que se produzca este proceso.

Bases funcionales y neurales del reconocimiento emocional. La localización de las áreas cerebrales relacionadas con el proceso de reconocimiento facial de emociones ha conllevado la realización de experimentos y estudios clínicos. En este sentido se destacan las investigaciones de Adolphs, Damasio, Tranel y Damasio (1996), quienes luego de estudiar a personas con lesiones en ambos hemisferios cerebrales encontraron que no existe deterioro en el procesamiento de expresiones faciales emocionales en los sujetos con lesiones únicamente en el hemisferio izquierdo, solo los daños en el hemisferio derecho se asocian a este deterioro. Otro resultado importante de esta investigación es que la mayoría de los daños se localizan en dos regiones de la neocorteza derecha: el córtex parietal inferior derecho de la superficie lateral y la corteza infracalcarina anterior en la superficie mesial. 
En posteriores estudios se señala la participación de las cortezas somatosensoriales en relación al reconocimiento de emociones, siendo necesaria para que el sujeto pueda generar una representación interna, que permitiría simular cómo otro puede sentirse al estar manifestando una determinada expresión emocional (Adolphs, Damasio, Tranel, Cooper \& Damasio, 2000).

A través del análisis de la potencia espectral de las diferentes bandas de frecuencia de la actividad cerebral, se observó un aumento de las ondas theta, simultáneo al incremento de las demandas atencionales y/o la dificultad de una tarea. También se ha encontrado que durante la elaboración de un estímulo emocional, en esta misma banda de frecuencia, se producía una mayor sincronización en la actividad hemisférica derecha y un aumento de la actividad de tipo delta. Estos hallazgos pudieran indicar que sólo estas bandas de frecuencia son sensibles al contenido emocional de un rostro (Fernández, Dufey \& Mourgues, 2007).

Investigaciones más recientes (Cereceda, Pizarro, Valdivia, Ceric, Hurtado, e Ibáñez, 2010), apuntan que la utilización de potenciales evocados (ERP), ha permitido la identificación de cambios breves en la actividad electroencefalográfica que ocurre ante la presencia de estímulos emocionales de rostros y palabras, y además se han podido detectar las áreas específicas de la corteza en las que estos ocurren. Mediante esta vía se ha probado que existen procesos cerebrales que se relacionan con el reconocimiento de emociones lateralizadas en el hemisferio derecho frente a rostros y en el hemisferio izquierdo ante palabras con contenido emocional.

El empleo del ERP por Bentin, Allison, Puce, Perez, y McCarthy (1996) ha permitido la descripción del curso temporal que sigue el procesamiento de rostros, de esta forma se ha encontrado que el componente más pronunciado de eventos relacionados a rostros corresponde a una polaridad negativa que se da en torno a los $170 \mathrm{~ms}$ después de la presentación del estímulo, o N170. En este sentido se señala además que a través de paradigmas experimentales que utilizan como estímulos la presentación de rostros expresando estados emocionales, se han reportado cambios en la actividad cerebral a los $100 \mathrm{~ms}$ y a los $250-500 \mathrm{~ms}$ después de la presentación de un estímulo.

De acuerdo con Fernández, Dufey y Mourguez (2007) estudios realizados han permitido concluir que el procesamiento de la expresión emocional precede al reconocimiento facial, esto deviene como resultado de investigaciones que demostraron la presencia de componentes más tempranos que el N170 que se dan a los 140-170, 120, e incluso a los $85 \mathrm{~ms}$ posteriores a la presentación del estímulo y que, además, son sensibles a la expresión emocional.

Se reconoce a través de exploraciones realizadas a pacientes con lesión cerebral, que la corteza orbitofrontal, la amígdala y el lóbulo temporal intervienen en el reconocimiento de las emociones (Sanz-Martín, Guevara, Corsi-Cabrera, OndarzaRovira \& Ramos-Loyo, 2006). En un estudio realizado a pacientes con prosopagnosia por Duchaine, Parker \& Nakayama (2003), se encontró posterior a la aplicación de seis test, problemas en el reconocimiento de la identidad facial, mientras que el reconocimiento de emociones en las pruebas aplicadas resultó normal.

Algunos autores como Kolb y Taylor (2000) se han enfocado en determinar la relación entre las estructuras cerebrales y emociones específicas. De esta forma señalan que las personas con lesiones en la región parietotemporal derecha presentan dificultades para reconocer expresiones faciales, fundamentalmente miedo y asco, y además manifiestan problemas para relacionar una cara con una caricatura que representa una escena afectiva. Adolphs (2008) reafirma esta idea y además enfatiza, basado en estudios de neuroimágenes, que las regiones ventrales de la corteza prefrontal se activan ante el miedo y la ira, por lo que el reconocimiento de estas emociones puede dañarse por las lesiones en esta región; y también las lesiones del estriado ventral pueden dañar el reconocimiento de la ira.

Se ha encontrado que el reconocimiento del asco evoca una respuesta en la ínsula anterior, en una región que probablemente también se asocia con procesar los olores y las sensaciones viscerales (Haxby, Hoffman \& Gobbini, 200). 
Adolphs et al. (1996) hallaron que pacientes con lesiones focales en el lóbulo parietal inferior derecho y en la corteza infracalcarina sobre la superficie mesial del hemisferio derecho tenían dificultades para reconocer las expresiones faciales de miedo y tristeza.

Muchos autores han discutido de la participación de la amígdala en el proceso emocional. La utilización de la resonancia magnética funcional ha revelado una activación considerable en la amígdala en respuesta a los rostros que expresan alegría y miedo. Sin embargo, los resultados de algunos estudios no son consistentes con esta noción, Wilson y Keil (1999) plantean que el reconocimiento de las emociones, incluidas el miedo, puede ocurrir incluso en ausencia de la amígdala y además las neuronas en la amígdala humana responden a las expresiones faciales particulares, pero exclusivamente a las expresiones de miedo.

Por otra parte, Fernández, et al., (2007) afirman que el daño bilateral en la amígdala produce una incapacidad en la fijación espontánea de la información proveniente de la región de los ojos, y por consiguiente afectaciones en el reconocimiento del miedo.

Una extensión importante del papel de la amígdala es a las enfermedades psiquiátricas en la que la relevancia conductual de estímulos se evalúa anormalmente. Enfermedades como las fobias, la depresión y la esquizofrenia han mostrado daños en el reconocimiento emocional y una activación anormal de la amígdala (Adolphs, 2008).

\section{Importancia del reconocimiento de emociones en las relaciones sociales}

Las emociones están presentes en todo momento de nuestra vida; nos relacionamos a través de ellas: experimentando alegría, ira, tristeza, sorpresa, miedo, asco, con diferente frecuencia y magnitud de acuerdo al contexto en el que surgen.

La posibilidad de reconocer emociones en los demás con precisión, modula nuestro comportamiento, de esta forma al interpretar correctamente las expresiones emocionales del rostro de una persona, se regula la conducta en función de una comunicación adecuada y de la interacción social positiva.
El reconocimiento exacto de expresiones faciales emocionales es un camino inicial para la respuesta empática. La empatía y el manejo de las relaciones interpersonales son necesarios desde el nacimiento para la supervivencia. La transmisión y reconocimiento de emociones entre madre e hijo son primordiales para generar un vínculo afectivo, esencial en la comunicación, pues las reacciones emocionales del neonato ayudan al adulto a entender sus necesidades.

Existe evidencia en estudios efectuados por Ekman (2004) de que quienes mejor ejercen el reconocimiento de las expresiones sutiles de la emoción están más abiertos a nuevas experiencias y suelen mostrar un mayor interés y curiosidad por las cosas, lo cual influye positivamente en el bienestar psicológico del individuo y su ambiente, pues una persona que puede verse más expuestas a mayores experiencias también podrá desarrollar mayormente sus capacidades sociales y las redes neuronales.

Cerceda, et al. (2010) señalan que una persona con menor capacidad de reconocer expresiones faciales difícilmente podrá entender las emociones asociadas a ella y el mundo emocional. La imposibilidad de contactarnos con nuestro estado afectivo o emocional puede alejarnos de nuestra capacidad de ser humanos, lo cual puede relacionarse con la aparición de enfermedades y patologías, que en su mayoría se encuentran ligadas a un no poder contactarnos con nosotros mismos y con nuestros propios sentimientos, así como también la realidad.

Alteraciones en el reconocimiento emocional en diversas patologías

Las personas que tienen afectaciones en el reconocimiento facial de emociones, por razones orgánicas como enfermedad u otras, también poseen afectadas significativamente otras esferas. Un ejemplo de ello es el área de las relaciones interpersonales: la aparente falta de empatía y la dificultad en la regulación del comportamiento, el cual puede parecer desajustado ante determinadas situaciones, limita el establecimiento y mantenimiento de estas relaciones (Cereceda et al., 2010).

En el campo de la neuropsicología existen investigaciones recientes que se han enfocado en 
determinar la posible relación entre algunas patologías y el proceso de reconocimiento de emociones faciales. Un estudio realizado por Cavieres y Valdebenito (2007) expuso la existencia de un déficit del reconocimiento de las emociones faciales en pacientes esquizofrénicos, que podría estar asociado con la disfunción cognitiva propia de la esquizofrenia. Los autores mencionan que se ha demostrado que estos pacientes tienen mayor dificultad para reconocer las emociones negativas, específicamente ira y miedo e interpretan de forma errónea las expresiones neutrales. Como un intento de explicación a este déficit en el reconocimiento se sugiere que reside en el análisis configuracional que realizan estas personas, pues no perciben los rostros de manera holística sino como una suma de partes.

Otra idea importante que señalan Cavieres y Valdebenito (2007) es que el problema en el reconocimiento de emociones se ha apreciado en casos de depresión, manía, lesiones cerebrales, demencia, autismo y específicamente en los pacientes con esquizofrenia existe una relación entre el estado clínico y la capacidad de reconocer las emociones faciales.

Adolphs, Tranel y Damasio (2001) manifiestan que, solamente los pacientes que sufren la lobectomía en el hemisferio derecho presentan una baja ejecución en el reconocimiento de las caras de miedo, sin que haya diferencias en el reconocimiento de la prosodia. Esto aporta evidencia sobre el efecto que puede acarrear la lobectomía, lo cual es congruente con el planteamiento de que cuando los pacientes tienen el foco epiléptico en el lóbulo temporal derecho pueden mostrar un deterioro en el reconocimiento de la identidad y de las expresiones faciales (Sanz-Martín et al., 2006).

Un estudio realizado en pacientes con la enfermedad de Parkinson, reveló la existencia de un déficit específico en el reconocimiento de emociones, lo que apunta a que en esta enfermedad pueden estarse produciendo distintas alteraciones cerebrales que se corresponden con problemas cognitivos y con las alteraciones en el reconocimiento afectivo (Alonso, Martín, Carvajal, Rubio, Ruis \& Serrano, 2012).
Las dificultades en la percepción de emociones son también comunes en la depresión y esto puede tener de base afectaciones cerebrales, probablemente en las áreas frontal ventral y las medio temporales. Los estudios de lesiones sugieren que la depresión es el resultado de las áreas límbica, paralímbica y prefrontal, lo que se relaciona con los informes de dificultades en la percepción de emoción facial en la depresión (Langenecker, Bieliauskas, Rapport, Zubieta, Wilde \& Berent, 2005).

El análisis de casos individuales ha reportado también evidencia de afectaciones en el reconocimiento de emociones. Un caso de demencia frontotemporal de predominio temporal derecho de siete años de evolución revela a través de la exploración neuropsicológica la existencia de alteraciones en la discriminación, emparejamiento, selección y denominación de emociones faciales especialmente negativas (ira, miedo, tristeza y asco); siendo más llamativa la afectación en los paradigmas de selección y denominación. Se señala que la emoción más afectada fue la ira y se valora la posibilidad de que la alteración del reconocimiento emocional podría estar en la base de determinadas alteraciones conductuales del paciente como el acercamiento a grupos marginales (García-Caballero, González-Hermida, García-Lado, \& Recimil, 2006).

Las investigaciones citadas demuestran que la habilidad para identificar expresiones faciales emocionales ha sido asociada con un rango amplio de psicopatologías, presentándose la sintomatología típica de cada alteración acompañada de un déficit en el reconocimiento emocional.

\section{Discusión}

La investigación relacionada con la memoria de rostros y el reconocimiento de emociones ha adquirido un gran impulso en la última década. El creciente interés por desentrañar las particularidades subyacentes en estos procesos ha tenido un importante avance a partir de la utilización integrada de herramientas psicológicas, neuroimágenes, pruebas neuropsicológicas y neurofisiológicas. Esta integración ha permitido que 
el conocimiento adquirido hasta el momento alcance una aplicabilidad práctica en diversos contextos de la vida cotidiana. La determinación de la veracidad del recuerdo de caras en testigos vinculados a procesos legales, el establecimiento de marcadores cognitivos para el diagnóstico temprano de enfermedades como el Alzheimer, la creación y desarrollo de programas computacionales para el reconocimiento automático de expresiones faciales, son algunas de las aplicaciones más sobresalientes e impactantes. No debe menospreciarse tampoco el importante papel en la comprensión de la empatía, la comunicación y el aprendizaje que el estudio de estos procesos ha impulsado. Sin embargo todavía existen innumerables preguntas sin respuestas en esta intrigante área del saber. Una de las interrogantes es sobre el papel que juega la experiencia en el desarrollo de la capacidad pare recordar rostros y reconocer emociones en las edades tempranas. Además se desconoce cómo se configuran los sistemas cognitivos en relación a estos mecanismos a lo largo de la maduración y de qué forma se reestructuran los procesos de memoria y reconocimiento de rostros luego de la ocurrencia de lesiones en el sistema nervioso central. Estas cuestiones necesitarán otras alternativas metodológicas, especialmente en relación a la creación de nuevas pruebas que evalúen ambos procesos y al mismo tiempo el diseño de estudios longitudinales. Lo que facilitará la elaboración de programas de rehabilitación de estas funciones, aspecto donde se evidencia en la actualidad una carencia importante.

No obstante, la psicología contemporánea (en especial la psicología cognitiva y la neuropsicología), continuará brindando una atención especial a los procesos que posibilitan la percepción de los rostros, su memorización y la decodificación de la información que muestra una cara.

\section{Referencias}

Adolphs, R. (2002). Recognizing Emotion From Facial Expressions: Psychological and Neurological Mechanisms. Behavioral and Cognitive Neuroscience Reviews, 1, 21-62.
Adolphs, R. (2008). Fear, Faces, and the Human Amygdala. National Institute of Health, 18(2), 166-172.

Adolphs, R., Damasio, H., Tranel, D., Cooper, G. \& Damasio, A. (2000). A role for the somatosensory cortices in the visual recognition of emotion as revealed by three dimensional lesion mapping. Journal of Neuroscience, 20, 2683-2690.

Adolphs, R., Damasio, H., Tranel, D. \& Damasio, A. (1996). Cortical Systems for the Recognition of Emotion in Facial Expressions. The Journal of Neuroscience 16(23): 7678-7687.

Adolphs, R., Tranel, D. \& Damasio, H. (2001). Emotion recognition from faces and prosody following temporal lobectomy. Neuropsychology, 15, 396-404.

Alonso, L., Martín, P., Carvajal, F., Rubio, S., Ruis, M.A. \& Serrano, J.M. (2012). Capacidad Cognitiva $y$ de Reconocimiento de Emociones en la Enfermedad de Parkinson. Madrid: Universidad Autónoma de Madrid.

Ardila, A. \& Ostrosky, F. (2012). Guía para el Diagnóstico Neuropsicológico. Florida: American Board of Professional Neuropsychology.

Ballesteros, S. (1999). Memoria humana: investigación y teoría. Psicothema, 11(4), 705-723.

Bänziger, T., Grandjean, D. \& Scherer, K. (2009). Emotion Recognition From Expressions in Face, Voice, and Body: The Multimodal Emotion Recognition Test (MERT). American Psychological Association, 9(5), 691-704.

Bentin, S., Allison, T., Puce, A., Perez, E. \& McCarthy, G. (1996). Electrophysiological studies of face perception in humans. Journal of Cognitive Neuroscience, 8 , 551-565.

Broche, Y. \& Herrera, L. F. (2011). Memoria de rostros y reconocimiento emocional: Particularidades en adultos mayores institucionalizados y que conviven con sus familiares. Revista Neuropsicologia Latinoamericana, 3(2), 12-19. 
Bruce, V. \& Young, A. (1986). Understanding face recognition. British Journal of Psychology, 77, 305-327.

Cavieres, A. \& Valdebenito, M. (2007). Déficit en el reconocimiento de emociones faciales en la esquizofrenia. Implicancias clínicas y neuropsicológicas. Revista Chilena de NeuroPsiquiatría, 45(2), 120-128.

Cereceda, S., Pizarro, I.,Valdivia, V., Ceric, F., Hurtado, E. \& Ibáñez, A. (2010). Reconocimiento de emociones: Estudio neurocognitivo. Praxis. Revista de Psicología, 2(18), 29-64.

Duchaine, B., Parker, H. \& Nakayama, K. (2003). Normal recognition of emotion in a prosopagnosic. Perception, 32, 827-838.

Duchaine, B. \& Yovel, G. (2008). Face recognition. The Senses: A Comprenhensive Reference, 2, 329-358.

Ekman, P. (2004). Emotions Revealed. New York: Times Books.

Fernández, A., Dufey, M. \& Mourgues, C. (2007). Expresión y reconocimiento de emociones: un punto de encuentro entre evolución, psicofisiología y neurociencias. Revista Chilena de Neuropsicología, 2, 8-20.

García-Caballero, González-Hermida, A., J., GarcíaLado I. \& Recimil M. I. (2006). Alteración en el reconocimiento de emociones faciales en un caso de demencia frontotemporal de predominio derecho. Actas Españolas de Psiquiatría, 34(6), 416-419.

García-García, R. \& Cacho-Gutiérrez, L. J. (2004). Prosopagnosia: ¿entidad única o múltiple? Revista de Neurología, 38(7), 682-686.

Gauthier, I., Behrmann, M.. \& Tarr, M. (1999). Can face recognition really de dissociated from object recognition? Cognitive Neuroscience, 11, 349-370.

Germine, L., Duchaine, B. \& Nakayama, K. (2010). Where cognitive development and aging meet: Face learning ability peaks after age 30. Cognition 118(2), 201-210.

Harris, A. \& Nakayama, K. (2007). Rapid face- selective adaptation of an early extrastriate component in MEG. Cortex, 17, 63-70.
Haxby, J. V., Hoffman, E. A. \& Gobbini, M. I. (2000). The distributed human neural system for face perception. Trends Cogn Sci, 4, 223-233.

Haxby, J. V., Hoffman, E. A. \& Gobbini, G. (2002). Human Neural Systems for Face Recognition and Social Communication. Society of Biological Psychiatry 51, 59-67.

Henson, R. N. A., Rugg, M. D., Shallice, T., Josephs, O., \& Dolan, R. (1999) Recollection and familiarity in recognition memory: an event-related fMRI study. Journal of Neuroscience, 19, 3962-3972.

Kandel, E. R. (2007). En busca de la memoria. Nacimiento de una nueva ciencia de la mente. Buenos Aires: Katz Editores.

Kanwisher, N., McDermott, J. \& Chun, M. (1997). The Fusiform Face Area: A Module in Human Extrastriate Cortex Specialized for Face Perception. The Journal of Neuroscience, 17(11), 4302-4311.

Kolb, B. \& Taylor, L. (2000). Facial expression, emotion and hemispheric organization. Cognitive neuroscience of emotion. New York: Oxford University Press.

Langenecker, S. A., Bieliauskas L. A., Rapport, L. J., Zubieta J. K., Wilde, E. A. \& Berent S. (2005). Face Emotion Perception and Executive Functioning Deficits in Depression. Journal of Clinical and Experimental Neuropsychology, 27(3), 320-333.

Levenson, R.W., Ascher, E., Goodkind, M., McCarthy, M., Sturm, V. \& Werner, K. (2008). Laboratory testing of emotion and frontal cortex. Handbook of Clinical Neurology, 88, 489-498.

Liu, J., Higuchi, M., Marantz, A. \& Kanwisher, N.(2002). Stages of processing in face perception: an MEG study. Nat. Neuroscience, 5, 910-916.

Liu, J., Higuchi, M., Marantz, A. \& Kanwisher, N. (2000). The selectivity of the occipitotemporal M170 for faces. Neuroreport, 11, 337-341.

Lopera, R. F. (2000). Procesamiento de caras: bases neurológicas, trastornos y evaluación. Revista de Neurología, 30(5), 1-5. 
Luria, A. R. (1982). El cerebro en acción. La Habana: Pueblo y Educación.

Manzanero, A. L. (2008). Procesos de recuperación en recuerdo y reconocimiento. Psicología del Testimonio. Madrid: Pirámide.

Manzanero, A. L., Grandes, I. \& Jódar, J. (2009). Edad y experiencia en el reconocimiento y la descripción de personas. Boletin de Psicología, 95, 87-98.

Molerio, O. (2004). Programa para el Autocontrol emocional de pacientes con hipertension arterial esencial, Universidad Central de Las Villas, Santa Clara.

Pozzulo, J. D. \& Lindsay, R. C. (1998). Identification accuracy of children versus adults: A meta-analysis. Law and Human Behavior, 22, 549-570.

Rolls, E. (1996). The orbitofrontal cortex. Phil Trans R Soc Lond, 351, 1433-1444.
Sanz-Martin, A., Guevara, M. A., Corsi-Cabrera, M., Ondarza-Rovira, R. \& Ramos-Loyo, J. (2006). Efecto diferencial de la lobectomía temporal izquierda y derecha sobre el reconocimiento y la experiencia emocional en pacientes con epilepsia. Revista de Neurología, 42(7), 391-398.

Thorpe, S. J., Rolls, E. T. \& Maddison, S. (1983). Neuronal activity in the orbitofrontal cortex of the behaving monkey. Exp Brain Res, 49, 93-115.

Wilson, R. A. \& Keil, F. C. (1999). The Mit Encyclopedia of the Cognitive Sciences. Massachusetts: Institute of Technology.

Yovel, G., Levy, J., Grabowecky, M. \& Paller, K. A. (2003). Neural correlates of the left-visual-field superiority in face perception appears at multiple stages of face perception. Cognitive Neuroscience, 15, 462-467.

Recibido: 25 de setiembre de 2013

Aceptado: 10 de febrero de 2014 